\title{
Uso de smartphones em viagens de turismo: análise do comportamento do mercado paulistano
}

\author{
Smartphones use in tourism trips: analysis of consumer behavior in São Paulo \\ (Brazil)
}

\author{
Jucylene Araújo dos Santos (SANTOS, J. A. dos) ${ }^{*}$ e \\ Glauber Eduardo de Oliveira Santos (SANTOS, G. E. de O.) ${ }^{* *}$
}

\begin{abstract}
RESUMO - Dentre as diversas novas tecnologias de comunicação que têm alterado a realidade do turismo, uma que merece grande destaque é o smartphone, aparelho de telefonia móvel que dispõe de uma série de recursos avançados. Além da possibilidade de realização de chamadas telefônicas praticamente a qualquer momento e em qualquer lugar, os smartphones oferecem inúmeras possibilidades de comunicação por meio da troca de informações digitais, incluindo textos, imagens, sons e vídeos. Esses aparelhos usualmente oferecem ainda outras facilidades, como câmera fotográfica e de vídeo, mídia player, jogos, leitores de texto, ferramentas de produtividade e outros aplicativos. $\mathrm{O}$ presente artigo tem como objetivo investigar o uso de smartphones durante viagens turísticas. Para tanto, foi realizada em setembro de 2013 uma pesquisa quantitativa por entrevistas diretas com 368 consumidores da cidade de São Paulo (Brasil) através da qual foram levantadas informações sobre os principais usos do aparelho em viagens, as formas de conexão utilizadas e os recursos preferidos desses viajantes. Além disso, analisou-se a satisfação dos usuários em relação à experiência de uso do dispositivo. Os resultados obtidos confirmaram que os smartphones são amplamente utilizados durante as viagens turísticas, apresentaram as funcionalidades mais utilizadas e apontaram novas oportunidades de interação com os consumidores para empresas e destinos turísticos.
\end{abstract}

Palavras-chave: Turismo e tecnologia; Turismo e comunicação; Smartphone; Comportamento do consumidor; São Paulo - SP.

ABSTRACT - Among several new communication technologies that have changed tourism reality, one which deserves attention is the smartphone. These mobile phones

\footnotetext{
* Formação: Graduação em Gestão de Turismo (Tecnologia) pelo Instituto Federal de Educação, Ciência e Tecnologia de São Paulo (IFSP). Atividade profissional: Coordenadora de Eventos na empresa Nascimento Promoções, Eventos e Incentivos. Endereço físico para correspondência: Rua Luis Carlos Peixoto, 50. CEP: 08430-410 - São Paulo (Brasil). Telefone para contato: (11) 97033-1278. E-mail: jucylene.araujo@gmail.com

** Formação: Graduação em Turismo (Bacharelado) pela Escola de Comunicações e Artes da Universidade de São Paulo (ECA-USP); Mestrado em Ciências da Comunicação - Lazer e Turismo pela ECA-USP; Mestrado e Doutorado em Economia do Turismo e do Meio Ambiente pela Universidade das Ilhas Baleares (Espanha) e Doutorando em Administração de Organizações pela Faculdade de Economia, Administração e Contabilidade de Ribeirão Preto da USP. Atividade profissional: Professor da área de Turismo e Hospitalidade do Instituto Federal de Educação, Ciência e Tecnologia de São Paulo (IFSP). Endereço físico para correspondência: Rua Pedro Vicente, 625 (Canindé). CEP 01109-010 - São Paulo São Paulo (Brasil). Telefone para contato: (11) 2763-7616. E-mail: glaubereduardo@ gmail.com
} 
improve communication not only by enabling calls anytime and anywhere, but also by offering alternatives for digital information exchange, including texts, images, sounds and videos. Besides communication, these gadgets' usually offer other features, such as photo and video cameras, media player, games, e-readers, productivity tools and other applications. This paper aimed to investigate the use of smartphones during tourist trips. In order to achieve that, a quantitative survey with 368 residents of São Paulo city was conducted in September 2013, gathering information about the main use of this device during tourism trips, the types of connections used and the preferred features of smartphones for travelers. Users' satisfaction experience of using smartphones during the trips was also analyzed. The results confirmed that smartphones are widely used during tourism trips and they presented the most used functionalities. New opportunities for companies and tourism destinations to interact with travelers were highlighted.

Key words: Tourism and technology; Tourism and communication; Smartphone; Consumer behavior; São Paulo (Brazil). 


\section{INTRODUÇÃO}

Turismo é uma atividade que exige grande trânsito de informações entre pessoas, empresas e outras entidades. Em especial, os turistas precisam de informação para escolher seus destinos e planejar suas viagens (BETTONI, 2009). Por isso, a relação entre turismo e novas tecnologias de comunicação sempre foi intensa. Conforme ressaltam Guimarães e Borges (2008), tecnologias inovadoras criam possibilidades de serviços inéditos e agregam valor a serviços já existentes.

Dentre as diversas novas tecnologias de comunicação que têm alterado a realidade do turismo, uma que merece grande destaque é a telefonia móvel. Os inúmeros benefícios dessa tecnologia vêm transformando a forma como os turistas se comunicam com outras pessoas e organizações. Além da possibilidade de realização de chamadas telefônicas praticamente a qualquer momento e em qualquer lugar, os telefones celulares mais modernos permitem também a comunicação por meio de outros sistemas. Os chamados smartphones ${ }^{1}$ oferecem inúmeras possibilidades por meio de troca de informações digitais, incluindo textos, imagens, sons e vídeos. Além da comunicação, esses aparelhos oferecem outras facilidades, como câmera fotográfica e de vídeo, mídia player, jogos, leitores de texto, ferramentas de produtividade e outros aplicativos.

A disseminação dos smartphones tem alterado substancialmente o comportamento dos indivíduos no mundo contemporâneo. De particular interesse para pesquisadores e gestores do turismo são as mudanças de comportamento dos indivíduos com respeito às viagens. As facilidades oferecidas por estes aparelhos trazem novas possibilidades de relacionamento do consumidor de turismo com destinos, empresas e pessoas. Contudo, as formas de uso de smartphones por parte dos turistas ainda são parcamente pesquisadas e conhecidas. Existe pouca informação disponível sobre como esses equipamentos têm alterado o comportamento do consumidor de turismo e quais são as implicações dessas mudanças para a gestão do setor. Desta forma, o presente trabalho tem como objetivo investigar o uso do smartphone durante as viagens dos turistas paulistanos. Para tanto, foi realizada pesquisa quantitativa por meio de 368

\footnotetext{
${ }^{1}$ Telefone inteligente, em tradução livre.
} 
entrevistas diretas com consumidores de viagens turísticas. $\mathrm{Na}$ seção a seguir se descreve o smartphone como ferramenta de comunicação e se apresenta detalhes do uso desses aparelhos. Na terceira seção se revisa a literatura existente sobre o uso dos smartphones em viagens turísticas. Em seguida são apresentadas a metodologia e os resultados do estudo deste levantamento. Por fim são apresentadas conclusões e recomendações.

\section{SMARTPHONES}

Os smartphones são aparelhos de telefonia móvel com funções avançadas, executadas ou até expandidas por meio de um sistema operacional ${ }^{2}$ (OXFORD DICTIONARIES ONLINE, 2013). Dentre as principais características desses equipamentos destacam-se a capacidade de conexão com redes de dados, o acesso à Internet, a maior capacidade de processamento de dados e a acessibilidade ampliada. Comparando os smartphones com outros aparelhos de telefonia móvel, nota-se a existência de muitas diferenças quanto à tela, processador e outros aspectos, as quais são resumidas no Quadro 1.

\section{QUADRO 1 - COMPARATIVO DOS TIPOS DE APARELHO CELULAR.}

\begin{tabular}{|llll|}
\hline CATEGORIA & \multicolumn{1}{c}{ ANALÓGICO } & \multicolumn{1}{c|}{ DIGITAL } & \multicolumn{1}{c|}{ SMARTPHONE } \\
\hline Formato & Tijolo & $\begin{array}{l}\text { Palm, concha do mar ou doce de } \\
\text { bar. }\end{array}$ & $\begin{array}{l}\text { Palm, concha do mar ou } \\
\text { doce de bar. }\end{array}$ \\
\hline Peso & $450 \mathrm{~g}$ a $900 \mathrm{~g}$ & $168 \mathrm{~g} \mathrm{a} \mathrm{225 \textrm {g }}$ & $<140 \mathrm{~g}$ \\
\hline Tela & Não disponível. & $\begin{array}{l}\text { Monocromático ou colorido, } \\
\text { pequeno, 172x120 pixels. }\end{array}$ & Colorido, 320 x 240 pixels. \\
\hline Processador & $\begin{array}{l}\text { Para funções básicas de } \\
\text { comunicação. }\end{array}$ & Para funções preliminares. & $\begin{array}{l}\text { Para funções avançadas, } \\
\text { como multimídia. }\end{array}$ \\
\hline Memória & $\begin{array}{l}\text { Apenas para estocar } \\
\text { números de telefones. }\end{array}$ & Alguns megabytes. & $\begin{array}{l}\text { 64 MB ou mais, adicional } \\
\text { SD Card flash. }\end{array}$ \\
\hline
\end{tabular}

Continua...

\footnotetext{
${ }^{2}$ Do inglês, Operating Systems (OS), são softwares que gerenciam diferentes programas e aplicações em dispositivos. Os mais populares para smartphones são: Android, IOS, Blackberry e Windows Phone.
} 
Continuação.

\begin{tabular}{|llll|}
\hline $\begin{array}{l}\text { Interfaces } \\
\text { compatíveis }\end{array}$ & Não disponível. & Sincronia com computadores. & Bluetooth, Wifi, GPS etc. \\
\hline Bateria & $\begin{array}{l}\text { Pequeno tempo de conversa } \\
\text { e tempo de espera. }\end{array}$ & $\begin{array}{l}\text { Longo tempo de conversa e tempo } \\
\text { de espera. }\end{array}$ & $\begin{array}{l}\text { Longo tempo de conversa e } \\
\text { tempo de espera. }\end{array}$ \\
\hline Preço & Alguns milhares de dólares. & $\begin{array}{l}\text { Uma vasta gama, de grátis a } \\
\text { algumas centenas de dólares. }\end{array}$ & $\begin{array}{l}\text { Algumas centenas de dólares } \\
\text { ou menos. }\end{array}$ \\
\hline
\end{tabular}

Fonte: Zheng e Ni (2006, p. 35, tradução nossa).

Diferentemente dos celulares analógicos e digitais, que são uma evolução direta da telefonia fixa, os smartphones têm origem também nos Personal Digital Assistants (PDAs). Dentre os PDAs mais populares, destacam-se os palmtops e os pocket PCs. De acordo com Zheng e Ni (2006), em sua essência, o smartphone surgiu da convergência entre celulares e PDAs.

Segundo Kim, Park e Morrison (2008) é possível destacar quatro características únicas quanto à natureza da tecnologia móvel presente nos smartphones. São elas:

- Ubiquidade: implica em quase onipresença, uma vez que o usuário pode acessar o dispositivo de qualquer lugar, buscando qualquer informação ou aplicação disponível.

- Personalização: cada usuário consegue a informação ou serviço que melhor lhe atende, de forma a ter disponível apenas as ferramentas que lhe convêm.

- Flexibilidade: possibilidade de realizar outras atividades enquanto se utiliza o dispositivo.

- Disseminação: graças às conexões, é possível compartilhar informações e dados com inúmeros outros usuários.

Os smartphones foram desenvolvidos e inseridos no mercado global no final dos anos 1990, mas se tornaram populares somente na virada do século (ZHENG; NI, 2006). O relatório anual Ericsson Mobility Report (ERICSSON, 2013) apontou que em 2012 já haviam sido vendidos no mundo mais de 1,1 bilhão de aparelhos e que este número tendia a crescer ainda mais nos próximos anos. Uma pesquisa realizada pela empresa Nielsen mostrou que a presença desses aparelhos é mais expressiva em países como Coréia do Sul, China, Austrália, Itália e Reino Unido (THE NIELSEN COMPANY, 2013). De acordo com essa pesquisa, enquanto em 2012 os smartphones representavam 67\% dos telefones celulares na Coréia do Sul, no Brasil esse percentual era de apenas $36 \%$. 
Segundo a IDC Consultoria (2014), em 2013 foram vendidos mais de 35 milhões de smartphones no Brasil, 123\% a mais que no ano anterior. As vendas de smartphones no país representam $52 \%$ do mercado de telefones celulares. Segundo a consultoria, o aumento nas vendas se deve principalmente à redução do preço médio do aparelho, à diversificação de modelos por parte dos fabricantes e à maior aceitação deste dispositivo pelos canais de venda.

$\mathrm{O}$ acesso à Internet pelo smartphone é feito em diferentes momentos. Uma pesquisa do Ibope Nielsen Online revelou que $84 \%$ dos usuários de smartphones acessavam a Internet enquanto esperavam para serem atendidos em algum local (IBOPE NIELSEN ONLINE, 2013). Segundo essa pesquisa, outras ocasiões bastante propícias ao uso da Internet no smartphone são durante o transporte cotidiano e enquanto o início de um evento é aguardado. A pesquisa identificou ainda que parte dos usuários de smartphones acessava a Internet enquanto trabalhavam, no banheiro, ao assistir televisão ou conversar com amigos.

Os conteúdos acessados nos smartphones são diversos. Ainda de acordo com a pesquisa IBOPE Nielsen Online (2013), os serviços mais populares foram os e-mails, busca de informações e redes sociais. Serviços de notícias, mapas, música, serviços bancários, previsão do tempo, vídeos e jogos também foram conteúdos frequentemente acessados. Por fim, cabe destacar que a pesquisa apontou que $10 \%$ dos usuários de smartphones utilizavam seus aparelhos para acessar conteúdos específicos sobre viagens.

\section{SMARTPHONES DURANTE AS VIAGENS}

Novas tecnologias têm sido utilizadas a fim de atender às necessidades dos turistas, criando inúmeras oportunidades para empresas e destinos, seja na criação de novos produtos e serviços ou na agregação de valor aos já existentes. De acordo com Guimarães e Borges (2007, p. 10):

O setor do turismo é um grande incorporador de tecnologia, nos seus diversos segmentos, e o seu crescimento sempre depende da capacidade de inovação e do uso da tecnologia para melhoria de gestão, desenvolvimento de novos produtos, aperfeiçoamento da comunicação, otimização das experiências de viagens e personificação do atendimento. 
Os smartphones aliados à Internet proporcionam grande conveniência aos turistas. Com o uso de ambos é possível acessar informações a quase todo momento e em quase todos os lugares. Os benefícios do smartphone para os turistas podem ser ilustrados por uma pesquisa que apontou que apenas $25 \%$ das pessoas nos Estados Unidos estariam dispostas a abrir mão do seu dispositivo em troca de descontos de $25 \%$ na hospedagem (MARRIOT, 2014). De acordo com Wang e Fesenmaier (2013, p. 58, tradução nossa):

[...] o uso de smartphones pode transformar significativamente a experiência de viagem, alterando o plano de viagem, construindo e desconstruindo o sentido do turismo para o indivíduo, e reconfigurando as relações entre turistas, lugares e outros.

Os smartphones podem fornecer aos viajantes informações antes da viagem, durante o deslocamento e ao longo da estada no destino (LAMFUS et al., 2013; WANG; PARK; FESENMAIER, 2011). Algumas das ferramentas mais úteis durante as viagens são a facilidade de comunicação por meio de ligações e mensagens de texto (SMS), a capacidade de armazenar arquivos para leitura, a disponibilidade de jogos e a realização de buscas e outras atividades online. Além dessas, os smartphones também possibilitam a troca de informações e interação por meio de aplicativos específicos. Wang, Park e Fesenmaier (2011) afirmam que os smartphones ajudam os turistas de duas formas distintas. Em algumas ocasiões os aparelhos contribuem na solução de problemas inesperados que ocorrem durante a viagem. Em outras eles auxiliam na realização de tarefas de forma mais eficaz e eficiente.

Wang e Fesenmaier (2013) notam que o uso do smartphone durante uma viagem permite que o indivíduo realize com maior facilidade algumas atividades rotineiras, tais como ouvir música, jogar e assistir vídeos. Segundo esses autores, essa possibilidade torna a viagem menos aborrecida. Por outro lado, os autores destacam que os smartphones permitem que os viajantes permaneçam conectados com o trabalho e outras obrigações, o que pode resultar em perda de qualidade da experiência de viagem. Wang e Fesenmaier ressaltam também que o smartphone amplia as possibilidades de socialização dos turistas durante suas viagens, o que provavelmente contribui com uma experiência positiva. 
Os aplicativos do segmento de viagens oferecem os mais diversos serviços, incluindo auxiliar o usuário a fazer as malas, registrar despesas, calcular custos de transporte, fazer reservas, traduzir textos e sons, obter informações de voos e muito mais. Uma pesquisa do TripAdvisor (2013b) mostrou que as tarefas mais frequentemente realizadas durante as viagens dos turistas norte-americanos com auxílio do smartphone são procurar restaurantes, acessar e postar conteúdos em redes sociais, buscar atividades parar fazer, ler opiniões de outros usuários e procurar hotéis.

Wang e Xiang (2012) ressaltam que os aplicativos de viagem de maior sucesso são aqueles que aproveitam os diferenciais do smartphone para oferecer ao usuário experiências mais ricas e especiais. Os autores afirmam que os usuários geralmente preferem aplicações que estão disponíveis somente nos smartphones, como aquelas que oferecem informações customizadas de acordo com a localização do usuário. Outro exemplo de aplicativo característico dos smartphones são aqueles que realizam funções à medida que o aparelho é chacoalhado. Por outro lado, os autores defendem que os usuários de smartphones tendem a perceber menor valor nos aplicativos que servem apenas como portão de entrada para websites comuns ou que apresentam programação pouco confiável.

A quantidade de aplicativos para smartphones disponíveis aos usuários tem crescido rapidamente a fim de satisfazer a demanda também em crescimento. $\mathrm{O}$ número total de aplicativos disponíveis no mercado atingiu a marca de um milhão em dezembro de 2011 (FREIERMAN, 2011). Menos de dois anos depois, em julho de 2013 a loja Google Play, que disponibiliza aplicativos para o sistema Android, atingia sozinha a mesma marca (WARREN, 2013). A loja Apple Store do sistema IOS alcançou um milhão de aplicativos pouco tempo depois, em dezembro de 2013 (JONES, 2013). No primeiro trimestre de 2014 a Google Play apresentava cerca de $45 \%$ mais downloads que a Apple Store, ainda que a receita desta última fosse $85 \%$ superior a da primeira (APP ANNIE, 2014). O Brasil se destacava como o segundo país com maior número de downloads na Google Play. Além disso, a categoria "Turismo e Local" constava como a quinta mais lucrativa dessa loja.

O Tripbarometer apontou que $51 \%$ dos viajantes mundiais costumam viajar com dispositivos móveis habilitados para uso de Internet (TRIPADVISOR, 2013a). A pesquisa apontou também que $33 \%$ dos viajantes usuários de smartphones utilizavam 
seus aparelhos para encontrar atividades locais nos destinos visitados. Outro dado bastante relevante dessa pesquisa indicou que $25 \%$ dos negócios turísticos no mundo já se relacionavam com clientes por meio de dispositivos móveis. A pesquisa aponta também que $72 \%$ dos viajantes no mundo indicaram que a opção de agendar e reservar hotéis através do dispositivo é bastante útil. Estar conectado por meio de um dispositivo móvel durante uma viagem é comum também no Brasil. Em média, 59\% dos viajantes brasileiros mencionaram utilizar dispositivos móveis habilitados para o uso de Internet durante suas viagens (TRIPADVISOR, 2013a).

\section{METODOLOGIA}

A fim de investigar o uso do smartphone durante viagens, para o presente artigo se desenvolveu pesquisa por meio de entrevistas quantitativas com paulistanos consumidores de viagens e usuários de smartphones. Ao todo foram entrevistados 368 indivíduos selecionados no Terminal Rodoviário Tietê, um importante ponto de fluxo de pessoas da cidade de São Paulo. As entrevistas foram realizadas em setembro de 2013 por alunos do curso de Tecnologia em Gestão de Turismo do Instituto Federal de Educação, Ciência e Tecnologia de São Paulo. A seleção de potenciais entrevistados foi realizada de forma acidental, sendo abordados praticamente todos os turistas presentes no local durante a realização do levantamento. Foram entrevistadas apenas pessoas que residiam na cidade de São Paulo, realizaram ao menos uma viagem nos doze meses anteriores ao momento da entrevista e que possuíam smartphone durante a viagem realizada.

Após a realização do levantamento de dados, os questionários passaram por uma análise crítica de consistência e confiabilidade. Os questionários inconsistentes ou pouco confiáveis foram descartados. A amostra final foi de 359 questionários. Foram levantadas informações sobre as preferências dos turistas, seus hábitos de viagem e uso dos smartphones durante as viagens realizadas.

A fim de alcançar resultados representativos do mercado paulistano como um todo, reduzindo-se o efeito de seleção da amostra resultante da realização do levantamento em um ponto específico da cidade, a pesquisa contou com um processo 
amostral estratificado por faixas etárias e classes socioeconômicas. Para a definição de classes foi utilizado o Critério de Classificação Econômica Brasil da Associação Brasileira de Empresas de Pesquisa (ABEP, 2012). A distribuição de smartphones na população por classes socioeconômicas foi estimada a partir da pesquisa da The Nielsen Company (2013). Essa pesquisa apontou que a maior concentração desses aparelhos no Brasil está na classe A (59\% dos usuários), seguida das classes B, C e D com 26\%, 11\% e $4 \%$, respectivamente.

A distribuição de smartphones na população por faixas etárias foi estimada a partir da combinação de informações do Censo Demográfico realizado em 2010 pelo Instituto Brasileiro de Geografia e Estática (IBGE, 2010) e da pesquisa The Mobile Consumer: A global snapshot (THE NIELSEN COMPANY. 2013). Esta última pesquisa identificou que a maior taxa de adesão ao smartphone ocorre na faixa etária de 16 a 24 anos. De acordo com os resultados, cerca de $41 \%$ dos indivíduos nessa faixa etária possuíam esse tipo de aparelho. Esses dados foram combinados com a distribuição da população paulistana por faixa etária dada pelo IBGE, considerando-se que ambas as distribuições são independentes.

Por fim, as distribuições dos smartphones por classes socioeconômicas e por faixa etária foram combinadas a fim de estimar a distribuição conjunta da população segundo esses dois critérios. Nessa combinação também se considerou que as duas distribuições eram independentes. O resultado, conforme apresentado na Tabela 1, aponta para que a maior ocorrência de smartphones se dava no grupo de classes A entre 25 a 34 anos.

TABELA 1 - DISTRIBUIÇÃO DA POPULAÇÃO QUE POSSUI SMARTPHONE SEGUNDO A CLASSE SOCIOECONÔMICA E A FAIXA ETÁRIA

\begin{tabular}{lccccc}
\hline \multirow{2}{*}{ Faixa etária } & \multicolumn{4}{c}{ Classe socioeconômica } & \multirow{2}{*}{ Total } \\
\cline { 2 - 5 } & $\mathbf{A}$ & $\mathbf{B}$ & $\mathbf{C}$ & $\mathbf{D}$ & \\
\hline 16 a 24 anos & $15 \%$ & $7 \%$ & $3 \%$ & $1 \%$ & $25 \%$ \\
25 a 34 anos & $19 \%$ & $8 \%$ & $3 \%$ & $1 \%$ & $32 \%$ \\
35 a 44 anos & $14 \%$ & $6 \%$ & $3 \%$ & $1 \%$ & $24 \%$ \\
45 a 64 anos & $11 \%$ & $5 \%$ & $2 \%$ & $1 \%$ & $19 \%$ \\
\hline Total & $\mathbf{5 9 \%}$ & $\mathbf{2 6 \%}$ & $\mathbf{1 1 \%}$ & $\mathbf{4 \%}$ & $\mathbf{1 0 0 \%}$ \\
\hline
\end{tabular}

Fonte: Cálculos dos autores, 2013.

O levantamento de dados buscou espelhar a distribuição da população apresentada na Tabela 1. Uma vez que os entrevistados foram selecionados acidentalmente, especial esforço teve que ser empregado para a obtenção de amostras 
não nulas em cada um dos cruzamentos possíveis. Como resultado deste processo, foram entrevistados 368 indivíduos distribuídos segundo suas classes socioeconômicas e faixas etárias conforme apresentado na Tabela 2.

TABELA 2 - DISTRIBUIÇÃO DA AMOSTRA DA PESQUISA

\begin{tabular}{|c|c|c|c|c|c|c|c|c|}
\hline \multirow{3}{*}{ Faixa etária } & \multicolumn{8}{|c|}{ Classe socioeconômica } \\
\hline & \multicolumn{2}{|c|}{$\mathbf{A}$} & \multicolumn{2}{|c|}{ B } & \multicolumn{2}{|c|}{$\mathbf{C}$} & \multicolumn{2}{|c|}{ D } \\
\hline & Amostra & $\%$ & Amostra & $\%$ & Amostra & $\%$ & Amostra & $\%$ \\
\hline 16 a 24 anos & 17 & $4,7 \%$ & 84 & $23,4 \%$ & 40 & $11,1 \%$ & 1 & $0,3 \%$ \\
\hline 25 a 34 anos & 19 & $5,3 \%$ & 96 & $26,7 \%$ & 41 & $11,4 \%$ & 1 & $0,3 \%$ \\
\hline 35 a 44 anos & 6 & $1,7 \%$ & 20 & $5,6 \%$ & 12 & $3,3 \%$ & 1 & $0,3 \%$ \\
\hline 45 a 64 anos & 6 & $1,7 \%$ & 11 & $3,1 \%$ & 3 & $0,8 \%$ & 1 & $0,3 \%$ \\
\hline
\end{tabular}

Fonte: Pesquisa de campo, 2013.

A divisão da distribuição estimada da população da pesquisa (Tabela 1) pela distribuição da amostra realizada (Tabela 2) resultou em pesos, os quais foram atribuídos a cada questionário com o objetivo de tornar a amostra representativa da população paulistana que possuía smartphone. Os pesos utilizados são apresentados na Tabela 3.

\section{TABELA 3 - PESOS}

\begin{tabular}{lllll}
\hline \multirow{2}{*}{ Faixa etária } & \multicolumn{4}{l}{ Classe socioeconômica } \\
\cline { 2 - 5 } & A & B & C & D \\
\hline 16 a 24 anos & 3,1 & 0,3 & 0,2 & 3,6 \\
25 a 34 anos & 3,5 & 0,3 & 0,3 & 4,5 \\
35 a 44 anos & 8,4 & 1,1 & 0,8 & 3,4 \\
45 a 64 anos & 6,8 & 1,6 & 2,5 & 2,8 \\
\hline
\end{tabular}

Fonte: Cálculos dos autores, 2013.

\section{RESULTADOS}

Segundo os resultados do levantamento, a maior parte dos paulistanos usuários de smartphones adotava o sistema operacional Android (71\%). O sistema IOS era utilizado por $27 \%$ dos indivíduos, enquanto outros sistemas, como Symbian e Blackberry, representavam $2 \%$ dos casos. A utilização de diferentes sistemas operacionais variou de acordo com a classe socioeconômica do indivíduo. Identificou-se que o sistema IOS estava sendo relativamente mais utilizado pelos indivíduos da classe 
C. Dentre as pessoas dessa classe socioeconômica, $41 \%$ utilizavam o IOS, enquanto nas classes A e B esse sistema operacional era adotado por apenas $26 \%$ dos indivíduos.

A grande maioria dos proprietários de smartphones mencionou utilizar seus aparelhos quando viajava (93\%). Dentre os indivíduos que não faziam este uso, a maioria não utilizava o aparelho regularmente nem mesmo quando não estavam viajando. Os indivíduos que indicaram não fazer uso do smartphone durante suas viagens apresentaram diferentes motivos para tal. Alguns indicaram não utilizar seus aparelhos como uma forma de se "desligar" das obrigações cotidianas. Outros fatores apontados foram o custo para utilizar serviços de dados e voz, a qualidade desses serviços em alguns locais, a duração da bateria e a preferência por outros dispositivos, como notebooks e tablets.

$\mathrm{Na}$ pesquisa se identificou que $93 \%$ dos paulistanos usuários de smartphones acessavam a Internet por meio do aparelho e $86 \%$ faziam esse uso diariamente. Quando em viagem, a taxa de utilização da Internet foi praticamente a mesma (94\%). Diferentes formas de acesso à Internet estavam sendo utilizadas de acordo com a disponibilidade do sinal ou preferência de cada indivíduo. A forma de acesso à Internet mais utilizada de acordo com a pesquisa era a rede de telefonia móvel. Os resultados apontaram que $86 \%$ dos paulistanos se valiam desse método. O uso do Wi-Fi também se revelou bastante comum, sendo adotado por $81 \%$ dos indivíduos.

$\mathrm{Na}$ pesquisa se levantou a taxa de utilização durante as viagens dos principais recursos oferecidos pelos smartphones. Os resultados estão apresentados na Figura 1. 


\section{FIGURA 1 - UTILIZAÇÃO DE RECURSOS DO SMARTPHONE DURANTE VIAGENS}

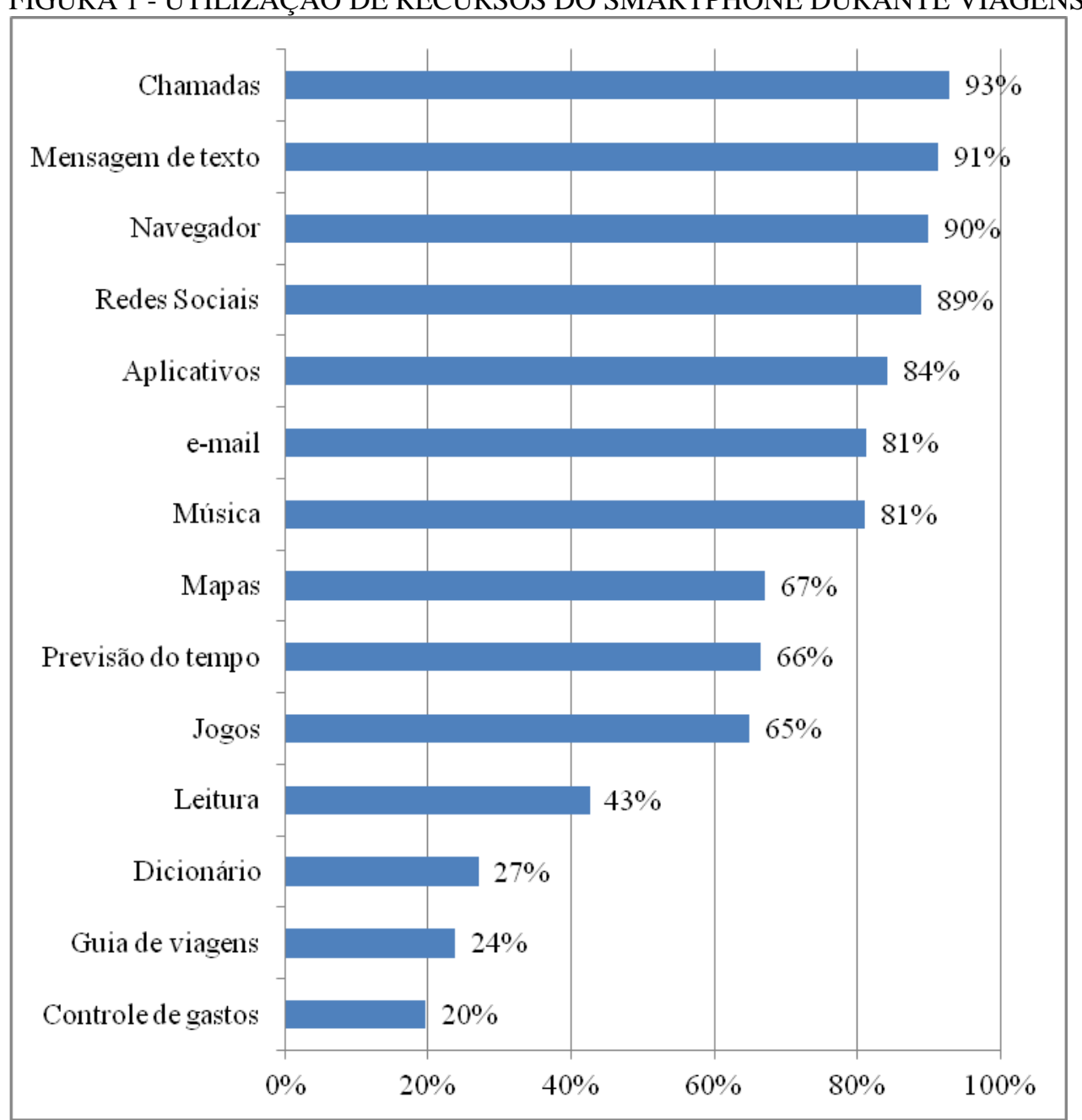

Fonte: Pesquisa de campo, 2013

As chamadas telefônicas enviadas e recebidas se constituíram no recurso mais empregado durante as viagens, sendo utilizado por $93 \%$ dos turistas. Mensagens de texto, navegador de Internet e redes sociais também se constituíram em aplicações bastante frequentes. Considera-se pertinente observar que as redes sociais se constituíram na ferramenta online de comunicação interpessoal mais utilizada, superando o e-mail, apesar de este último ser um recurso mais antigo e tradicional. Dentre os recursos menos utilizados ficaram os aplicativos de controle de gastos, guias de viagens e dicionários.

Na pesquisa se investigou também qual o nível de satisfação dos indivíduos com os resultados do uso do smartphone em viagens. As informações coletadas mostraram que $88 \%$ dos entrevistados ficaram satisfeitos ou muito satisfeitos com a experiência. Quando questionados sobre o motivo da satisfação, as principais respostas foram a 
respeito da comodidade proporcionada pelo dispositivo e solução de problemas. Por outro lado, os principais motivos apresentados para a insatisfação estavam relacionados a problemas de conexão, dificuldade para localizar a informação ou serviço de interesse, tempo gasto com a busca, ineficiência do aparelho e ao fato de algumas páginas não estarem disponíveis em formato compatível com a tecnologia de telefonia móvel.

\section{CONSIDERAÇÕES FINAIS}

O uso de smartphones tem se tornado uma prática comum entre os turistas. A constante presença deste dispositivo e suas comodidades modificam o comportamento dos viajantes em diferentes aspectos. Essa nova realidade cria oportunidades para empresas e destinos oferecerem novos produtos e agregarem valor aos antigos.

Nesta pesquisa se descreveu o comportamento de uso dos smartphones pelos turistas do maior mercado consumidor do Brasil. Os resultados apontaram que a grande maioria dos paulistanos com smartphones estava utilizando o aparelho também quando viajava. Além disso, a pesquisa mostrou que esse uso estava sendo frequente e com diversificados objetivos. Desta forma, comprovou-se a proposição de que o smartphone é um importante meio de comunicação para os turistas.

Dentre as diferentes funcionalidades oferecidas pelos smartphones aos turistas, as mais utilizadas foram as chamadas telefônicas, mensagens de texto, navegação na Internet, redes sociais, tocadores de áudio e e-mails. Mapas, previsão do tempo e jogos também se constituíram em aplicações bastante utilizadas. Por outro lado, dicionários, guias de viagens e sistemas de controle de gastos apresentaram utilização relativamente pequena. Considera-se que essas informações são particularmente úteis para subsidiar a análise dos investimentos feitos por empresas e órgãos gestores de destinos turísticos no desenvolvimento aplicações de tecnologia mobile. Investimentos em aplicativos com baixa diferenciação e que não fazem uso das características especiais dos aparelhos móveis podem atrair poucos usuários e apresentar em pequeno retorno. Por outro lado, aplicações inovadoras, integradas com as funcionalidades mais utilizadas e que despertem a curiosidade dos turistas podem oferecer bons resultados para empresas e destinos turísticos. Nesse conjunto, uma alternativa que apresenta grande potencial de 
contribuição para a experiência turística é a Realidade Aumentada, tecnologia que amplia o entorno do indivíduo por meio de informações virtuais integradas com o ambiente físico (YOVCHEVA; BUHALIS; GATZIDIS, 2012).

A presente pesquisa limitou-se a analisar o uso dos smartphones por parte dos turistas paulistanos. Pesquisas com foco geográfico mais abrangente ou que tratem dos turistas de outras localidades podem ser de grande valia para análises da possibilidade de generalização dos resultados aqui apresentados. Além disso, deve-se destacar que a realidade aqui tratada é extremamente dinâmica, de modo que parte dos resultados apresentados é altamente perecível. Por fim, sugere-se também que pesquisas futuras abordem o uso dos smartphones em viagens por meio de métodos qualitativos, buscando-se maior aprofundamento sobre questões ligadas às motivações, sentimentos e satisfação dos usuários.

\section{REFERÊNCIAS}

ABEP - Associação Brasileira de Estudos Populacionais. Critério de Classificação Econômica Brasil. São Paulo: ABEP, 2012.

APP ANNIE. App Annie Index - Market Q1 2014: revenue soars in the United States and China. Disponível em: <http://blog.appannie.com/app-annie-index-market-q12014>. Acesso em: 17/04/2014.

BETTONI, E. M. A colaboração dos usuários na divulgação de destinos turísticos baseada no compartilhamento de dados geoprocessados. Turismo \& Sociedade, v. 2, n. 1, p. 64-89, 2009.

ERICSSON AB. Ericsson Mobility Report: On The Pulse Of The Networked Society: February 2013. Disponível em: <http://www.ericsson.com/ericsson-mobility-report>. Acesso em: 26/05/2013.

FREIERMAN, S. One million mobile apps, and counting at fast pace. The New York Times, 11 dez. 2011. Disponível em: <http://www.nytimes.com/2011/12/12/technology/one-million-apps-andcounting.html>. Acesso em: 17/04/2014.

GUIMARÃES, A. S.; BORGES, M. P. E-turismo: Internet e Negócios do Turismo. São Paulo: Cengage Learning. 2008. 
IBGE. Instituto Brasileiro de Geografia e Estatística. Censo Demográfico 2010. 2010. Disponível em: <http://www.censo2010.ibge.gov.br>. Acesso em: 10/06/2013.

IBOPE NIELSEN ONLINE. Smartphone é companhia constante dos brasileiros. Disponível em:

$<$ http://www.ibope.com/pt-br/relacionamento/imprensa/releases/Paginas/Smartphonee-companhia-constante-dos-brasileiros.aspx >. Acesso em: 03/05/2013.

IDC CONSULTORIA. Estudo da IDC aponta que mercado brasileiro de celulares encerrou 2013 com a marca recorde de 67,8 milhões de unidades comercializadas. 2014. Disponível em: <http://br.idclatin.com/releases/news.aspx?id=1613>. Acesso em: 17/04/2014.

JONES, C. Apple's app store about to hit 1 million apps. Forbes, 12 dez. 2013. Disponível em: <http://www.forbes.com/sites/chuckjones/2013/12/11/apples-app-storeabout-to-hit-1-million-apps/>. Acesso em: 17/04/2014.

KIM, D.; PARK, J.; MORRISON, A. A model of traveller acceptance of mobile technology. International Journal of Tourism Research, v. 10, p. 393-407, 2008.

LAMFUS, C.; XIANG, Z.; ALZUA-SORZABAL, A.; MARTÍN, D. Conceptualizing context in an intelligent mobile environment in travel and tourism. In: CANTONI, L.; XIANG, Z. (Orgs.). Information and Communication Technologies in Tourism 2013. Heidelberg: Springer, 2013. p. 1-11.

MARRIOT. SpringHill Suites Annual Travel Survey. 2013. Disponível em: <http://news.marriott.com/springhill-suites-annual-travel-survey.html>. Acesso em: 17/04/2014.

OXFORD DICTIONARIES ONLINE. 2013. Oxford University press. Disponível em: <http://oxforddictionaries.com/definition/english/smartphone>. Acesso em: 10/06/2013.

THE NIELSEN COMPANY. The Mobile Consumer: A Global Snapshot. Disponível em: <http://www.slideshare.net/duckofdoom/mobile-consumerreport2013-17748641>. Acesso em: 16/05/2013.

TRIPADVISOR. TripBarometer. 2013a. Disponível em: <http://www.tripadvisortripbarometer.com>. Acesso em: 16/05/2013.

TripBarometer Mobile \& Social Survey Finds 85\% of US Travelers Bring Their Smartphones on Vacation \& 61\% Report Using Social Media While Traveling. 2013b. Disponível em:

$<$ http://www.tripadvisor.com/TripAdvisorInsights/n2006/tripbarometer-mobile-socialsurvey-finds-85-us-travelers-bring-their-smartphones-vacation-61-report>. Acesso em: 17/04/2014. 
YOVCHEVA, Z.; BUHALIS, D., GATZIDIS, C. Overview of smartphone augmented reality applications for tourism. E-Review of Tourism Research, v. 10, n. 2, p. 63-66, 2012.

WANG, D.; FESENMAIER, D. R. Transforming the travel experience: the use of smartphones for travel. In: CANTONI, L.; XIANG, Z. (Orgs.). Information and Communication Technologies in Tourism 2013. Heidelberg: Springer, 2013. p. 5869.

WANG, D.; PARK, S.; FESENMAIER, D. The role of smartphones in mediating the touristic experience. Journal of Travel Research, v. 51, n. 4, p.1-17, 2011.

WANG, D.; XIANG, Z. The new landscape of travel: a comprehensive analysis of smartphone apps. In: FUCHS, M.; RICCI, F.; CANTONI, L. (Orgs.). Information and Communication Technologies in Tourism 2012. Heidelberg: Springer, 2012. p. 308319.

WARREN, C. Google Play hits 1 million apps. Mashable, 24 jun. 2013. Disponível em: <http://mashable.com/2013/07/24/google-play-1-million/>. Acesso em: 17/04/2014.

ZHENG, P.; NI, L. Smart Phone and Next Generation Mobile Computing. São Francisco: Morgan Kufmann, 2006.

Recebido em: 28-04-2014.

Aprovado em: 28-05-2014. 